\title{
CLINICAL FEATURES OF TOXIC JAW BONE OSTEOMYELITIS IN DRUG ADDICTS
}

DOI:10.36740/WLek202102116

\author{
Oleh S. Fitkalo', Roman Z. Ohonovskyi' , Khrystyna R. Pohranychna', Yaroslav P. Nahirnyi², Andriy V. Netlyukh' \\ 'DANYLO HALYTSKY LVIV NATIONAL MEDICAL UNIVERSITY, LVIV, UKRAINE \\ 2IVAN HORBACHEVSKYI TERNOPIL NATIONAL MEDICAL UNIVERSITY, TERNOPIL, UKRAINE
}

\begin{abstract}
The aim: The aim of our research is to study the features of toxic osteomyelitis in drug addicts, their diagnosis and comprehensive treatment, aimed at strengthening motivation for the suspension of the use of psychoactive substances and the elimination of the pathological process in the lower jaw .

Materials and methods: The features of toxic osteomyelitis, complicated by abuse, have been studied on 46 patients in the department of oral and maxillofacial surgery of Lviv regional clinical hospital during 2013-2019. Psychoactive substances, used by the patients, varied from homemade drugs "Screw" taken by 32 men (69.5\%), synthetic drug amphetamine consumed by 10 men $(21,7 \%)$ to Subutex used by 4 patients $(8,7 \%)$ for their pleasure. All the patients underwent comprehensive examination, which included clinical, laboratory, radiological, pathohistological studies. Almost all patients - 41 ( $89.1 \%$ ) underwent sequestrectomy.

Results: Clinical picture progressed quite rapidly in the form of diffuse destructive-necrotic osteomyelitis of the mandible, which was characterized by a severe, atypical course of the pathological process with permanent inclusion of other additional areas of the lesion of the mandible. The $X$-ray at this stage showed an increase in destructive processes in the bone.

Conclusions: Appropriate surgical tactics and pathogenetic therapy are of great importance for toxic osteomyelitis in drug addicts. Due to the treatment, despite the total destruction of the mandible, it was possible to stop the destructive bone processes and to preserve life for such patients.
\end{abstract}

KEY WORDS: drug abuse, toxic osteomyelitis, Jaw bones

Wiad Lek. 2021;74(2):263-267

\section{INTRODUCTION}

A huge problem of modern medicine is a holistic approach to treating a patient, taking into account all the comorbid conditions resulting from an unhealthy lifestyle (abuse of psychoactive substances ) and social disadvantage. At present, abuse has become one of the most serious global problems, caused by crisis of morality, culture, change of life values, and loss of sense of life, mostly affecting young people. All this, unfortunately, has led to sad statistics in Ukraine which holds the "palm of primacy" in Europe in terms of psychoactive substance use. According to official data alone (the Ministry of Health of Ukraine 2019 report on people with mental disorders due to the use of all groups of psychoactive substances) in Ukraine, more than 519 thousand persons are being monitored, with 452 thousand of them having mental disorders and behavior due to alcohol consumption, and $40-42 \%$ of patients having comorbid pathology. One of the diseases, which often occurs during drug abuse, is toxic osteomyelitis of bones. The factual material, covered in considerable detail in literature sources [ $1,2,3$, 4 ], enables us to detail the clinical picture of this serious disease. According to the authors V.O.Malanchuk and , I.S.Brodetsky $(2009,2010,2013,2014)$ the total number of patients with drug abuse is $60 \%$ of the total amount of patients with osteomyelitis of the jaws without comorbid pathology. The authors studied etiology, pathogenesis, clinical pictures and the ways to overcome the disease. It was revealed that the patients have atypical occurrence of odontogenic processes against the background of drug addiction with serious long course, frequent relapses, rapid spread of the pathological process, low efficiency of traditional treatment regimens, which is directly connected with a lengthy use of drugs. The effects of a prolonged use of psychoactive substances are rejection, sequestration of parts of the jaws, loss of natural teeth $[5,6,7]$. The process of damaging the bone tissue of jaws sometimes lasts for months or years. At the same time the patient completely rejects the fact that it is the substance abuse that has such serious consequences. Only a small percentage of patients think about the cause , which has led to such a condition, and refuse from psychoactive substance abuse, which helps to stop the development of the pathological process in the jaw .

\section{THE AIM}

The aim of our research is to study the features of toxic osteomyelitis in drug addicts, their diagnosis and comprehensive treatment, aimed at strengthening motivation for the suspension of the use of psychoactive substances and the elimination of the pathological process in the lower jaw . 


\section{MATERIALS AND METHODS}

To study the features of toxic osteomyelitis, complicated by abuse, 46 patients of the department of oral and maxillofacial surgery of the regional clinical hospital in Lviv were examined during the period from 2013 to 2019. The average age of the patients ( men ) was $26.5 \pm 6.57$. In all patients, the pathological process was localized in the mandibular area. Twenty-nine patients (63.0\%) people, the majority of patients, took drugs for more than 5 years; 15 (32.6\%) patients took drugs for not more than two years. The patients mainly consumed hard drugs or homemade drugs for up to two years, which is quite harmful for health .

The clinical picture of toxic osteomyelitis in psychoactive substance addicts directly depended on the duration of abuse and the composition of drugs. In 10 ( $21.7 \%$ ) patients with long-term use of psychoactive substances $>5$ years, the pathological process progressed rapidly due to dense and thick cortical plates, which hindered the break of pus on the outer surface of the bone, with high intoxication and damage to new parts of the jaw .

Having given their consent, all patients underwent comprehensive examination, which included clinical, laboratory, radiological, pathohistological studies. When collecting the history from patients, it was found that all of them consumed drugs. All this was very important for further treatment, because the patients tried to conceal that they took drugs and were addicts. Psychoactive substances, used by the patients, varied from homemade drugs "Vint" taken by 32 men (69.5\%), synthetic drug amphetamine consumed by 10 men $(21,7 \%)$ to Subutex used by 4 patients $(8,7 \%)$ for their pleasure .

All patients underwent complex treatment with surgical intervention and subsequent medication, with establishing contact between doctor and patient. The effectiveness of the treatment depended on many factors such as age of patients, the duration psychoactive substance abuse, the condition of the patient upon arrival and others.

Almost all patients - 41 ( $89.1 \%$ ) underwent sequestrectomy, after which the prescription of antibiotics was not the only drug treatment, since patients with osteonecrosis of the jaws with drug addiction have deep pathological changes in various systems of the body (immune, cardiovascular, hematopoietic, hemostatic). All other patients did not require surgery, since the destruction of bone tissue (according to the results of CT) was not accompanied by the formation of sequesters, or their sizes were insignificant, and they came out through the fistula.

Moreover, in the patients with pathogenic addiction, the microflora is highly resistant to antibiotics. Therefore, the combined use of systematic enzymotherapy (namely wobenzym ) and intramuscular administration of clindamycinum resulted in a high concentration of the antibiotic in pyo-necrotic focus of the bone tissue. Clindamycinum has an immune modulating property and is a derivative of lincosamides, effectively used to treat osteomyelitis. We have proposed the introduction of $5 \mathrm{ml}$ clindamycinum intramuscularly 2 times a day for 10 days. Clindamycin should be used with caution in patients with severe hepatic impairment. In our research group, severe liver pathology was not observed in either instrumental or clinical studies.

With severe pain, 32 ( 69.6\% ) of 46 patients were offered nimesulide, included in the group of non - steroidal anti - inflammatory drugs, 1 tab. 3 times a day to reduce pain within 5 days. All 46 patients were prescribed wobenzym, 1 tab. 3 times a day. It consists of highly active enzymes of plant and animal origin and has anti-inflammatory, anti-edematous effect and fibrinolytic effect, which allowed to normalize the immune imbalance and to increase the therapeutic effectiveness of antibiotics. The therapeutic effect of the drug is aimed at accelerating the lysis of toxic products, which contributed to the improvement of microcirculation .

Given the complex comorbid pathology, the integrated approach to medical treatment was extremely relevant and was directed at improving vital parameters by using the drugs that eliminate, first of all, immune imbalance, and enhance the overall condition of the body. At the beginning of treatment, for detoxification, all patients were prescribed $15 \mathrm{mg}$ enterosgel 3 times a day for 14 days. The narcologist, in his turn, prescribed $24 \mathrm{mg}$ polioksidony three times a day. This drug has a clearly pronounced immuno modulating action, detoxification and antioxidant activity, removes toxins and salts of heavy metals from the body, helps to inhibit peroxide oxidation of lipids, which provides an increasing resistance of the organism to the local and generalized infections. Twenty-nine $(63.0 \%)$ patients, who were in a state of withdrawal syndrome remission, were prescribed hidazepam $50 \mathrm{mg} 3$ times a day sublingually. After taking the drug that has a mild tranquilizing and anxiolytic effect with antidepressant components,

psychomotor agitation, anxiety and irritability almost did not appear.

For a better understanding of the problem of the features of toxic osteomyelitis in addicts, we fully described the entire clinical picture of comorbid pathology and its treatment on the example of one of our patients who came to the clinic after the occurrence of edema of admaxillary soft tissue edema. The patient at once confirmed the longterm use of psychoactive substances ( 4,5 years ), which allowed avoiding relapse in the postoperative period, using an integrated approach to treatment.

\section{RESULTS}

Most of the patients who participated in our study used hard or artisanal drugs for at least two years. Of the whole group, 4 participants $(8.7 \%)$ used Subutex. When collecting the anamnesis, it was found that these patients used subutex as a drug intravenously for four years. The condition of these patients in terms of the severity of the concomitant pathology, toxic osteomyelitis, did not differ from the patients who abused other drugs, which did not contradict our study.

The toxicity of various impurities of homemade drugs (such as free iodine) lead to necrotic-degenerative processes in bone tissue, which is the cause of osteomyelitis and total bone destruction. 


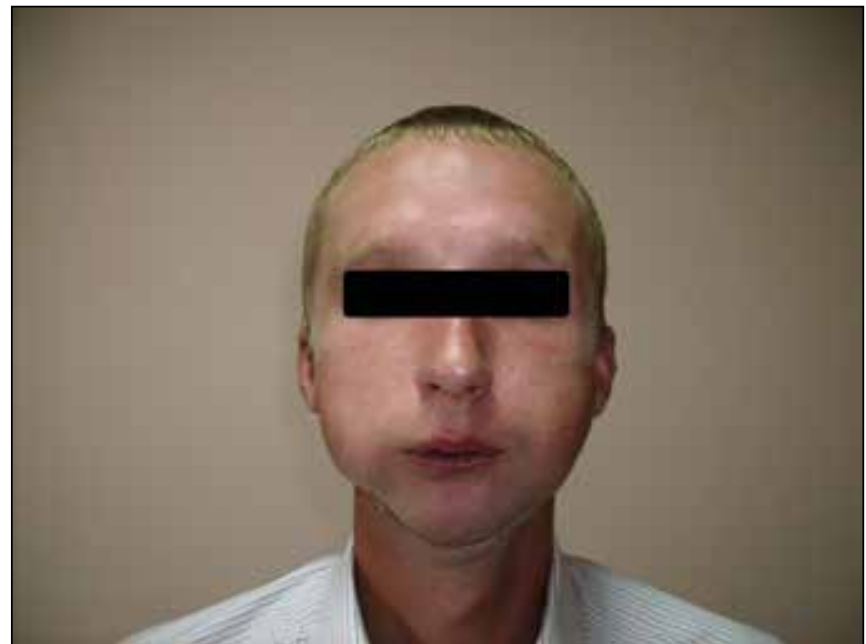

Fig 1. Photograph of the patient in the frontal projection at the end of diffuse osteomyelitis in the lower jaw: there is a decline of soft facial tissues in its lower third

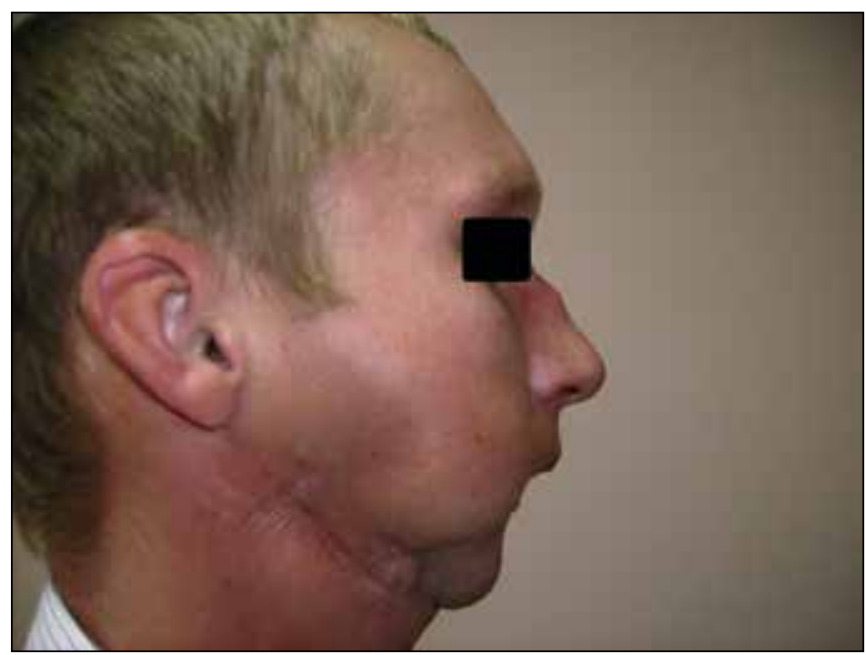

Fig 2. Photograph of the same patient in lateral projection: in addition to soft tissue sinking, in the upper cervical area, there are scars from multiple surgical interventions.

During the first clinical examination, an asymmetric face was observed in the patient due to swelling and infiltra- tion of the soft tissues. These phenomena were known to have occurred after exacerbation of chronic periodontitis of 46 tooth and its removal. The patient indicated that he has many years of experience in the use of psychotropic substances of the primary series.

In the following days, with the spread of the inflammatory process, the patient felt pain on palpation of the relevant parts of the bone, and a significant thickening of the jaw was visually observed. Prolonged intoxication provoked aching pains, which were associated with the appearance of new foci of osteomyelitis and relapses, as well as significant fistulas.

Further, the clinical picture progressed quite rapidly in the form of diffuse destructive-necrotic osteomyelitis of the mandible, which was characterized by a severe, atypical course of the pathological process with permanent inclusion of other additional areas of the lesion of the mandible. Several times after the formation of bone sequesters, the patient underwent sequestrectomy surgery and was prescribed appropriate treatment, which included not only a combination of surgical, medication and orthopedic methods, but also consultation of a physician-narcologist and physician-psychotherapist for further treatment of addiction. (Figs. 1, 2).

The X-ray at this stage showed an increase in destructive processes in the bone. The final orthopantomogram showed total destruction and complete absence of the chin, body and angles of the mandible and, partially, up to $1 / 2$ the height of the branches of the mandible on both sides. On the cult of the mandibular branch to the left there is a radiological shadow of the reconstructive plate, which was used as an immobilizing agent of the jaw fragments, which were further lysed (Fig. 3).

\section{DISCUSSION}

Research into literature sources indicated that by the conscious domestic use of psychotropic substances, Ukraine is one of the countries where it makes up a significant percentage, and the problem itself is becoming more and more social, especially among the younger generation $[1,2]$

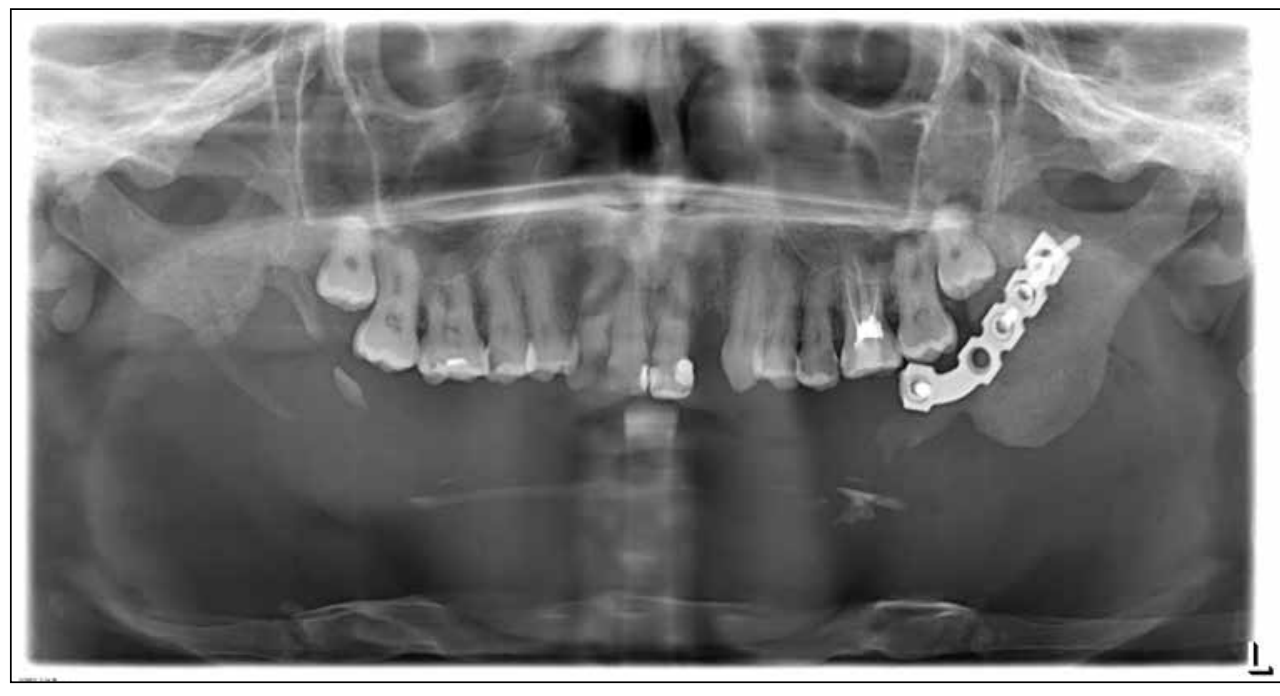

Fig 3. Orthopantogram of a patient with many years of experience in the use of narcotic drugs of the primary series. 
Our observations, which completely coincide with other scientific studies, reveal that up to $40-42 \%$ of such adherents also have various comorbid pathologies, which significantly complicates their treatment and social adaptation $[3,4]$.

Working in the Department of Maxillofacial Surgery of LNMU, we have recently found a significant increase in diffuse toxic osteomyelitis of jaw bones, which is also confirmed by the data of the studied scientific works, and their percentage reaches $60 \%[2,7]$. Analysis of case histories of such patients showed an atypical course of odontogenic processes against the background of drug dependence with a severe long-term course, frequent relapses, rapid spread of the pathological process, low efficiency of traditional treatment regimens, which is directly related to long-term drug use. The consequences of long-term use of psychoactive substances are rejection, sequestration of jaw parts, loss of natural teeth $[5,6,7]$. The process of damage to the jaw bone tissue sometimes lasts for months or years. At the same time, the patient completely rejects the fact that the very abuse of psychoactive substances has such serious consequences [3].

Conducting this study, the authors set the task to study the peculiarities of toxic osteomyelitis in drug addicts, their diagnosticss and comprehensive treatment, which aims to increase motivation to suspend the use of psychoactive substances and eliminate the pathological process in the lower jaw.

To study the peculiarities of toxic osteomyelitis, complicated by drug abuse, 46 patients of the Department of Maxillofacial Surgery of the Lviv Regional Clinical Hospital were examined during the period from 2013 to 2019. The average age of patients (men) was $26.5 \pm 6.57$. In all patients, the pathological process was localized in the lower jaw. Twenty-nine (63.0\%) persons, who made up the majority of patients, had been on drugs for more than five years; 15 (32.6\%) people had taken drugs for no more than two years.

The clinical picture of toxic osteomyelitis in people who abuse psychoactive substances directly depended on the duration of use and their composition. Thus, in $10(21.7 \%)$ patients with long-term use of psychoactive substances $>5$ years, the pathological process developed rapidly due to dense and thick cortical plates, which made it difficult for pus to break through to the outer surface of bone, with high intoxication and damage to new parts of jaws.

All patients underwent comprehensive treatment with surgery and subsequent medical treatment with the contact established between doctor and patient. Almost all patients - $41(89.1 \%)$ persons underwent sequestration, after which, given that patients with jaw osteonecrosis against the background of drug dependence have profound pathological changes in various body systems (immune, vascular, hematopoietic, hemostasis), medical treatment was not limited to the prescription of antibiotics. Moreover, in drug dependent patients, pathogenic microflora has high antibiotic resistance.
Therefore, systemic enzyme therapy (namely, wobenzyme) and administration of clindamycin, used simulnaneously, provided a higher concentration of antibiotic in the purulent-necrotic focus of bone tissue. We proposed the intramuscular administration of Clindamycin 2 times a day for $5 \mathrm{ml}$ for 10 days. In case of severe pain, 32 (69.6\%) patients out of 46 were offered Nimesulide, which belongs to the group of nonsteroidal anti-inflammatory drugs, 1 tab. 3 times a day for 5 days to reduce pain. All 46 patients were prescribed Wobenzyme, 1 tab. 3 times a day, which includes highly active enzymes of plant and animal origin with anti-inflammatory, anti-edematous, fibrinolytic effect, which made it possible to normalize the immune imbalance, increase the therapeutic efficacy of antibiotics. The therapeutic effect of the drug is aimed at accelerating the lysis of toxic metabolic products, which improved microcirculation.

Given the complex comorbidity, a comprehensive approach to drug treatment was essential and aimed at improving vital signs with the use of drugs that eliminate, first of all, immune imbalance, and strengthen the general condition of the body. At the beginning of treatment, for detoxification, all patients were prescribed Enterosgel, 15 mg 3 times per day for 14 days.

The narcologist, in turn, offered the patients $24 \mathrm{mg}$ of Polyoxidonium 3 times per day. The drug has a pronounced immunomodulatory effect, detoxifying and antioxidant activity, removes toxins, salts of heavy metals, and inhibits lipid peroxidation, which increases the body's resistance to local and generalized infection. Twenty-nine (63.0\%) patients who were in remission of the withdrawal syndrome were prescribed Gidazepam, $50 \mathrm{mg} 3$ times a day, sublingually. After taking the drug, which has a mild tranquilizing and anxiolytic effect with antidepressant components, psychomotor agitation, anxiety and irritability were almost non-existent.

We believe that the obtained positive results allow us to consider this set of measures as the one that reduces the percentage of disability of patients with a stable refusal to abuse psychoactive substances and allows saving the life of such patients and create conditions for subsequent reconstructive surgery.

\section{CONCLUSIONS}

Appropriate surgical tactics and pathogenetic therapy are of great importance for toxic osteomyelitis in drug addicts. Therefore, in our study, with long-term integrated treatment, systemic and pathogenetically conditioned drug therapy was used, and appropriate surgical treatment, aimed at removal of necrotized areas of the mandibular bone, was performed. We believe that due to the treatment, despite the total destruction of the mandible, it was possible to stop the destructive bone processes and to preserve life for such a patient and to create conditions for subsequent reconstructive operations. The treatment was aimed at reducing the disability of patients with the persistent rejection of substance abuse. 


\section{REFERENCES}

1. Malanchul V.A., Brodetskyi I.S., Zabudskaya L.R. Radioghraphic peculiarities of the lower jaw osteomyelities in patients with drug addiction. Ukrainian Medical Journal. 2009;2:122-125.

2. Malanchul V.A., Brodetskyi I.S. Osteomyelities of the jaws in patients with drug addiction. 2013:268.

3. Malanchul V.A., Brodetskyi I.S. Comprehensive treatment of patients with jaw osteomyelitis with drug dependence. Herald of VSMU. 2014;13(2):115-123.

4. Timofeyev A.A. Clinical features and surgical treatment of primary odontogenic inflammatory lesions in patients with purulentinflammatory diseases of the soft tissues using drugs «Screw». Modern dentistry. 2010;3:121-127.

5. Chernov D.V., Komok 0.A., ShmatS.M. et al. Some aspects of the jawbone osteomyelitis treatment in individuals with immunodeficiency states. Medical perspectives. 2010;15(2):1-3.

6. Saberov R.Z., Drobyshev A.Yu. Jaw necrosis in people with immunodeficiency while taking drugs. New Technologies in Dentistry: XVI International Conference of Oral and Maxillofacial Surgeons: Materials of Conf.SPb, 2011:157-158.

7. Ruzin H.P. Clinical and radiological variants of the facial bones osteomyelitis course in drug-dependent patients. Ukrainian Dental Almanac. 2013;1:46-50.

\section{ORCID and contributionship:}

Oleh S. Fitkalo: 0000-0001-6321-9518 ${ }^{A, C}$

Roman Z. Ohonovskyi: 0000-0003-0959-0863 ${ }^{A, C}$

Khrystyna R. Pohranychna: 0000-0002-3366-0799 D, F

Yaroslav P. Nahirnyi: 0000-0002-1530-0271 ${ }^{E}$

Andriy V. Netlyukh:0000-0002-6617-0438 ${ }^{B}$

\section{Conflict of interest:}

The Authors declare no conflict of interest.

\section{CORRESPONDING AUTHOR Khrystyna R. Pohranychna \\ Danylo Halytsky Lviv National Medical University \\ 69 Pekarska Str., 79010 Lviv, Ukraine \\ tel: +380987762355 \\ e-mail:pohranychna@ukr.net}

Received: 27.03 .2020

Accepted: 23.10 .2020

A - Work concept and design, B - Data collection and analysis, C - Responsibility for statistical analysis,

D-Writing the article, $\mathbf{E}$-Critical review, $\mathbf{F}$ - Final approval of the article 